\title{
Editorial
}

\section{Novel Structured Catalytic Reactors}

\author{
Gianluca Landi (D)
}

Citation: Landi, G. Novel Structured Catalytic Reactors. Catalysts 2021, 11, 1472. https://doi.org/10.3390/ catal11121472

Received: 16 November 2021 Accepted: 19 November 2021 Published: 1 December 2021

Publisher's Note: MDPI stays neutral with regard to jurisdictional claims in published maps and institutional affiliations.

Institute of Sciences and Technologies for Sustainable Energy and Mobility-CNR, 80125 Naples, Italy; gianluca.landi@cnr.it

Structured catalytic reactors are widely used in the automotive sector for exhaust aftertreatment, thus representing the state-of-art technology in this sector. A structured reactor is generally composed of a mechanical support with a defined geometry and a washcoat layer (few microns) is deposited onto the support walls. The washcoat is (or supports) the active phase. Honeycomb monoliths and foams are the most used mechanical supports. The success of structured catalysts is due to their low pressure drops, high thermal and mechanical stability and, in case of fast reactions, better utilization of the active phase due to a reduced thickness of the active layer, i.e., a short diffusion distance within the catalyst (low Thiele modulus, high catalyst efficiency). Accordingly, structuring of the catalyst and reactor is a successful approach in processing intensification. As a matter of fact, structured catalysts allow higher rates and selectivities by reducing mass and heat transport limitations. Moreover, operation within the flammability limits can be carried out safely; as a result, characteristic channel width is generally lower than the quenching distance of fuels. Due to the easy scaling (up and down), small installation can be designed favoring the decentralization of production and the reduction of transportation costs.

Additionally, the features of the mechanical support can significantly affect the overall performance of the catalytic reactor. For instance, monoliths with high thermal conductivity can be used to flatten the temperature profiles of a high exothermic or endothermic reaction, thus improving both performance (higher average temperatures) and durability (few hot spots).

In recent decades, several efforts have been made in order to realize more and more efficient structured reactors and to extend their use to novel applications. For instance, packed foams were used to improve heat transfer with respect to packed beds in a reactor, characterized by high volumetric catalyst density. Another example is represented by catalytic microreactors, which are intrinsically structured catalysts. They offer excellent mass and heat transfer performance, and the simple coupling of exothermal and endothermal reactions. Due to low flow rates through each channel, small throughput is obtained in each channel; however, by the so-called numbering-up approach, simpler than the scalingup approach, the capacity of microchannel reactors can be rapidly increased. Advanced structured catalytic reactors also require the development of novel preparation techniques. Among others, a special mention is to give to 3D printing and electro-deposition.

A key feature of the structured catalytic reactors is the co-occurrence of multiple chemical and physical phenomena (mass transfer to, inside, and from the catalytic layer, radial and axial heat transfer, chemical reaction on the catalyst surface) related not only to the intrinsic features of the active phase, but also to substrate properties. In this respect, several phenomena have been understood and/or previewed by numerical studies, especially by CFD simulations.

This Special Issue reports some examples on the recent advancements in the development of novel structured catalytic reactors proposed for very different applications. Thus, in the following, a brief description of the papers included in this issue is provided to serve as an outline to encourage further reading.

Power to Gas (PtG) is a promising solution for the long-term storage of surplus electric energy. Electric power can be converted into hydrogen, and further converted 
with captured $\mathrm{CO}_{2}$ into synthetic methane. In [1], the aging of a structured $\mathrm{Ni} / \mathrm{Al}_{2} \mathrm{O}_{3}$ catalytic reactor is studied under real operating conditions as well as the reasons for the aging process. Long runs $(100 \mathrm{~h})$ were carried out and fresh and aged catalysts were characterized by $\mathrm{N}_{2}$ physisorption, X-ray diffractometry (XRD), and temperatureprogrammed oxidation (TPO). The Authors reported that no activity loss was observed at low temperatures $\left(<300{ }^{\circ} \mathrm{C}\right)$, while by increasing the temperature higher and higher, a decrease in the performance of the catalyst was detected, up to a 7\% drop in methane yield for the $500{ }^{\circ} \mathrm{C}$ test. The aging was correlated with a decrease in total surface area and, consequently, of the exposed active nickel surface.

Catalytic combustion is one of the classic applications of structured catalysts for both the removal of pollutants (unburnt compounds, carbon monoxide, organic wastes) and power generation. A current and well-known application is the abatement of $\mathrm{HC}$ and $\mathrm{CO}$ in the exhausts of diesel engines in the so-called diesel oxidation catalyst (DOC). In these applications, the use of structured catalytic reactors is mandatory due to a need for low pressure drops. In recent decades, a novel application of catalytic combustion has resulted in the development of micro-combustors, i.e., combustors with a characteristic radial dimension lower than the extinction length. It has been clearly reported in the scientific literature that catalytic, rather than homogeneous, micro-combustors offer stable operations in a wider range of operating conditions. However, the issues regarding the stability of catalytic micro-combustors are still up for debate. In [2], inspired by the efficient heat management of reindeer nasal geometry, Di Sarli et al. proposed a novel spiral catalytic micro-combustor and simulated its behavior using a three-dimensional CFD model. In particular, the authors investigated the quenching behavior of lean propane/air mixtures at different values of residence time (i.e., inlet gas velocity) and (external convective) heat transfer coefficient at fixed micro-combustor dimensions, comparing the obtained results with simulations of the same conditions for a standard parallel-channel configuration of equivalent dimensions. The two types of quenching phenomena (i.e., blow-out and extinction) were identified depending on the operating conditions. In particular, results in [2] showed that blow-out is not significantly affected by the geometrical configuration, while in the advanced configuration, extinction occurs at higher values of residence time and heat transfer coefficient, thus guaranteeing a wider operational range. The improved performance of the novel spiral catalytic micro-combustor, inspired by reindeer nasal geometry, is due to its ability to keep the heat generated using combustion trapped inside the micro-combustor.

A fundamental feature of structured catalytic reactors, significantly affecting their performance, is the relative ratio among intrinsic kinetics, internal mass transfer (i.e., diffusion inside the pores) and external mass transfer (i.e., mass transport between bulk fluid phase and external catalyst surface). Catalytic layers deposited onto structured supports are generally very thin; therefore, a fast transition from kinetic to external mass transfer limitation is commonly detected. In this issue, Gancarczyk et al. [3] studied the effect of the morphology on the heat and mass transfer and on the pressure drops of different metal foams, comparing their catalytic activity towards slow or fast reactions with honeycomb monoliths. Commercial foams made of aluminum, nickel, nickel-chromium, and FeCrAlloy, with pore density ranging from 10 to $40 \mathrm{PPI}$, were used. Under the investigated experimental conditions, heat and mass transfer in solid foams were much more intense than those of monoliths, and similar to those of packed beds. Accordingly, for slow reaction kinetics (as in the case of selective catalytic reduction of $\mathrm{NO}$ with $\mathrm{NH}_{3}$ ), when the mass transfer was not limiting, specific surface area (or catalyst amount) was the decisive factor. On the contrary, for fast reaction kinetics (as in the case of methane combustion), mass transfer became limiting and, for carriers of intense mass transfer, such as solid foams, the reactor length was much shorter than for the others, thus also positively affecting pressure drops due to reactor shortening. The authors also showed that the heat transfer rate between the catalyst surface and the bulk reactant stream may be insufficient depending on reaction features, such as the reaction enthalpy, and operating conditions, such as inlet reactants 
concentration and temperature. The selection of the proper catalyst carrier, with the most favorable transport parameters, can contribute to overcome this limitation.

An innovative application of structured catalytic reactors was proposed by Eliasz et al. [4] In their work, the authors proposed a catalytic dehydrogenation pre-treatment of the fuel to be injected into a self-ignition engine with the aim of both improving performance and lowering pollutant emissions. In order to realize the pre-treatment, the authors installed a modified common rail (CR) high-pressure battery containing a Pt-catalyzed rod with annular channels and a modified fuel injector atomizers with Pt-added spiral-elliptical channels on the nonworking part of the needle. Accordingly, the catalysts worked at a high pressure and the fuel was pre-treated just before being injected into the cylinders. Results showed that the presence of hydrogen in the injected fuel affected the combustion process; in particular, carbon monoxide and particulate emissions were slightly reduced, while NOx emissions were unaffected by fuel pre-treatment. Moreover, at a low engine load, i.e., at lower pressure in the system, the fuel flows moved more slowly through the atomizer channels and were pre-heated at higher temperatures. Higher fuel temperature and hydrogen presence in the feed stream can reduce the self-ignition delay period, thus improving the combustion efficiency.

Finally, as guest editor, I would like to thank the authors who contributed to this issue for all of their valuable contributions. I would also like to express my gratitude to all the staff in the editorial office of Catalysts, in particular to Ms. Caroline Zhan, for their support in the preparation and realization of this Special Issue.

Funding: There is no external funding.

Conflicts of Interest: The author declares no conflict of interest.

\section{References}

1. Morosanu, E.A.; Salomone, F.; Pirone, R.; Bensaid, S. Insights on a methanation catalyst aging process: Aging characterization and kinetic study. Catalysts 2020, 10, 283. [CrossRef]

2. Di Sarli, V.; Trofa, M.; Di Benedetto, A. A novel catalytic micro-combustor inspired by the nasal geometry of reindeer: Cfd modeling and simulation. Catalysts 2020, 10, 606. [CrossRef]

3. Gancarczyk, A.; Sindera, K.; Iwaniszyn, M.; Piatek, M.; Macek, W.; Jodłowski, P.J.; Wroński, S.; Sitarz, M.; Łojewska, J.; Kołodziej, A. Metal foams as novel catalyst support in environmental processes. Catalysts 2019, 9, 587. [CrossRef]

4. Eliasz, J.; Osipowicz, T.; Abramek, K.F.; Matuszak, Z.; Mozga, Ł. Fuel pretreatment systems in modern ci engines. Catalysts 2020, 10, 696. [CrossRef] 